\title{
A generalized metric space and related fixed point theorems
}

\author{
Mohamed Jleli and Bessem Samet ${ }^{*}$
}

${ }^{\text {*Correspondence: }}$

bsamet@ksu.edu.sa

Department of Mathematics,

College of Science, King Saud

University, P.O. Box 2455, Riyadh,

11451, Saudi Arabia

MSC: $54 \mathrm{H} 25 ; 47 \mathrm{H} 10$

Keywords: generalized metric; b-metric; dislocated metric; modular space; fixed point; partial order

\section{Introduction}

The concept of standard metric spaces is a fundamental tool in topology, functional analysis and nonlinear analysis. This structure has attracted a considerable attention from mathematicians because of the development of the fixed point theory in standard metric spaces.

In recent years, several generalizations of standard metric spaces have appeared. In 1993, Czerwik [1] introduced the concept of a $b$-metric space. Since then, several works have dealt with fixed point theory in such spaces; see [2-7] and references therein. In 2000, Hitzler and Seda [8] introduced the notion of dislocated metric spaces in which self distance of a point need not be equal to zero. Such spaces play a very important role in topology and logical programming. For fixed point theory in dislocated metric spaces, see [9-12] and references therein. The theory of modular spaces was initiated by Nakano [13] in connection with the theory of order spaces and was redefined and generalized by Musielak and Orlicz [14]. By defining a norm, particular Banach spaces of functions can be considered. Metric fixed theory for these Banach spaces of functions has been widely studied. Even though a metric is not defined, many problems in fixed point theory can be reformulated in modular spaces (see [15-20] and references therein).

In this work, we present a new generalization of metric spaces that recovers a large class of topological spaces including standard metric spaces, $b$-metric spaces, dislocated metric spaces, and modular spaces. In such spaces, we establish new versions of some known fixed point theorems in standard metric spaces including Banach contraction principle, Ćirićs fixed point theorem, a fixed point result due to Ran and Reurings, and a fixed point result due to Nieto and Rodíguez-López.

\begin{abstract}
We introduce a new concept of generalized metric spaces for which we extend some point theorem, a fixed point result due to Ran and Reurings, and a fixed point result due to Nieto and Rodríguez-López. This new concept of generalized metric spaces
recover various topological spaces including standard metric spaces, $b$-metric spaces, dislocated metric spaces, and modular spaces.
\end{abstract}

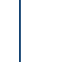




\section{A generalized metric space}

Let $X$ be a nonempty set and $\mathcal{D}: X \times X \rightarrow[0,+\infty]$ be a given mapping. For every $x \in X$, let us define the set

$$
C(\mathcal{D}, X, x)=\left\{\left\{x_{n}\right\} \subset X: \lim _{n \rightarrow \infty} \mathcal{D}\left(x_{n}, x\right)=0\right\} .
$$

\subsection{General definition}

Definition 2.1 We say that $\mathcal{D}$ is a generalized metric on $X$ if it satisfies the following conditions:

$\left(\mathcal{D}_{1}\right)$ for every $(x, y) \in X \times X$, we have

$$
\mathcal{D}(x, y)=0 \quad \Longrightarrow \quad x=y
$$

$\left(\mathcal{D}_{2}\right)$ for every $(x, y) \in X \times X$, we have

$$
\mathcal{D}(x, y)=\mathcal{D}(y, x)
$$

$\left(\mathcal{D}_{3}\right)$ there exists $C>0$ such that

$$
\text { if }(x, y) \in X \times X,\left\{x_{n}\right\} \in C(\mathcal{D}, X, x) \text {, then } \mathcal{D}(x, y) \leq C \limsup _{n \rightarrow \infty} \mathcal{D}\left(x_{n}, y\right) \text {. }
$$

In this case, we say the pair $(X, \mathcal{D})$ is a generalized metric space.

Remark 2.2 Obviously, if the set $C(\mathcal{D}, X, x)$ is empty for every $x \in X$, then $(X, \mathcal{D})$ is a generalized metric space if and only if $\left(\mathcal{D}_{1}\right)$ and $\left(\mathcal{D}_{2}\right)$ are satisfied.

\subsection{Topological concepts}

Definition 2.3 Let $(X, \mathcal{D})$ be a generalized metric space. Let $\left\{x_{n}\right\}$ be a sequence in $X$ and $x \in X$. We say that $\left\{x_{n}\right\} \mathcal{D}$-converges to $x$ if

$$
\left\{x_{n}\right\} \in C(\mathcal{D}, X, x) .
$$

Proposition 2.4 Let $(X, \mathcal{D})$ be a generalized metric space. Let $\left\{x_{n}\right\}$ be a sequence in $X$ and $(x, y) \in X \times X$.If $\left\{x_{n}\right\} \mathcal{D}$-converges to $x$ and $\left\{x_{n}\right\} \mathcal{D}$-converges to $y$, then $x=y$.

Proof Using the property $\left(\mathcal{D}_{3}\right)$, we have

$$
\mathcal{D}(x, y) \leq \limsup _{n \rightarrow \infty} \mathcal{D}\left(x_{n}, y\right)=0,
$$

which implies from the property $\left(\mathcal{D}_{1}\right)$ that $x=y$.

Definition 2.5 Let $(X, \mathcal{D})$ be a generalized metric space. Let $\left\{x_{n}\right\}$ be a sequence in $X$. We say that $\left\{x_{n}\right\}$ is a $\mathcal{D}$-Cauchy sequence if

$$
\lim _{m, n \rightarrow \infty} \mathcal{D}\left(x_{n}, x_{n+m}\right)=0 .
$$

Definition 2.6 Let $(X, \mathcal{D})$ be a generalized metric space. It is said to be $\mathcal{D}$-complete if every Cauchy sequence in $X$ is convergent to some element in $X$. 


\subsection{Examples}

In this part of the paper, we will see that this new concept of generalized metric spaces recovers a large class of existing metrics in the literature.

\subsubsection{Standard metric spaces}

Recall that a standard metric on a nonempty set $X$ is a mapping $d: X \times X \rightarrow[0,+\infty)$ satisfying the following conditions:

$\left(\mathrm{d}_{1}\right)$ for every $(x, y) \in X \times X$, we have

$$
d(x, y)=0 \quad \Longleftrightarrow x=y ;
$$

$\left(\mathrm{d}_{2}\right)$ for every $(x, y) \in X \times X$, we have

$$
d(x, y)=d(y, x)
$$

$\left(\mathrm{d}_{3}\right)$ for every $(x, y, z) \in X \times X \times X$, we have

$$
d(x, y) \leq d(x, z)+d(z, y)
$$

It is not difficult to observe that $d$ satisfies all the conditions $\left(\mathcal{D}_{1}\right)-\left(\mathcal{D}_{3}\right)$ with $C=1$.

\subsection{2 b-Metric spaces}

In 1993, Czerwik [1] introduced the concept of $b$-metric spaces by relaxing the triangle inequality as follows.

Definition 2.7 Let $X$ be a nonempty set and $d: X \times X \rightarrow[0,+\infty)$ be a given mapping. We say that $d$ is a $b$-metric on $X$ if it satisfies the following conditions:

$\left(b_{1}\right)$ for every $(x, y) \in X \times X$, we have

$$
d(x, y)=0 \quad \Longleftrightarrow \quad x=y
$$

$\left(\mathrm{b}_{2}\right)$ for every $(x, y) \in X \times X$, we have

$$
d(x, y)=d(y, x)
$$

$\left(\mathrm{b}_{3}\right)$ there exists $s \geq 1$ such that, for every $(x, y, z) \in X \times X \times X$, we have

$$
d(x, y) \leq s[d(x, z)+d(z, y)]
$$

In this case, $(X, d)$ is said to be a $b$-metric space.

The concept of convergence in such spaces is similar to that of standard metric spaces.

Proposition 2.8 Any b-metric on $X$ is a generalized metric on $X$. 
Proof Let $d$ be a $b$-metric on $X$. We have just to proof that $d$ satisfies the property $\left(\mathcal{D}_{3}\right)$. Let $x \in X$ and $\left\{x_{n}\right\} \in C(d, X, x)$. For every $y \in X$, by the property $\left(\mathrm{b}_{3}\right)$, we have

$$
d(x, y) \leq s d\left(x, x_{n}\right)+s d\left(x_{n}, y\right)
$$

for every natural number $n$. Thus we have

$$
d(x, y) \leq s \limsup _{n \rightarrow \infty} d\left(x_{n}, y\right)
$$

The property $\left(\mathcal{D}_{3}\right)$ is then satisfied with $C=s$.

\subsubsection{Hitzler-Seda metric spaces}

Hitzler and Seda [8] introduced the notion of dislocated metric spaces as follows.

Definition 2.9 Let $X$ be a nonempty set and $d: X \times X \rightarrow[0,+\infty)$ be a given mapping. We say that $d$ is a dislocated metric on $X$ if it satisfies the following conditions:

$\left(\mathrm{HS}_{1}\right)$ for every $(x, y) \in X \times X$, we have

$$
d(x, y)=0 \quad \Longrightarrow \quad x=y
$$

$\left(\mathrm{HS}_{2}\right)$ for every $(x, y) \in X \times X$, we have

$$
d(x, y)=d(y, x)
$$

$\left(\mathrm{HS}_{3}\right)$ for every $(x, y, z) \in X \times X \times X$, we have

$$
d(x, y) \leq d(x, z)+d(z, y)
$$

In this case, $(X, d)$ is said to be a dislocated metric space.

The motivation of defining this new notion is to get better results in logic programming semantics.

The concept of convergence in such spaces is similar to that of standard metric spaces.

The following result can easily be established, so we omit its proof.

Proposition 2.10 Any dislocated metric on $X$ is a generalized metric on $X$.

\subsubsection{Modular spaces with the Fatou property}

Let us recall briefly some basic concepts of modular spaces. For more details of modular spaces, the reader is advised to consult [19], and the references therein.

Definition 2.11 Let $X$ be a linear space over $\mathbb{R}$. A functional $\rho: X \rightarrow[0,+\infty]$ is said to be modular if the following conditions hold:

$\left(\rho_{1}\right)$ for every $x \in X$, we have

$$
\rho(x)=0 \quad \Longleftrightarrow \quad x=0
$$


$\left(\rho_{2}\right)$ for every $x \in X$, we have

$$
\rho(-x)=\rho(x)
$$

$\left(\rho_{3}\right)$ for every $(x, y) \in X \times X$, we have

$$
\rho(\alpha x+\beta y) \leq \rho(x)+\rho(y)
$$

whenever $\alpha, \beta \geq 0$ and $\alpha+\beta=1$.

Definition 2.12 If $\rho$ is a modular on $X$, then the set

$$
X_{\rho}=\left\{x \in X: \lim _{\lambda \rightarrow 0} \rho(\lambda x)=0\right\}
$$

is called a modular space.

The concept of convergence in such spaces is defined as follows.

Definition 2.13 Let $(X, \rho)$ be a modular space.

(i) A sequence $\left\{x_{n}\right\}_{n \in \mathbb{N}} \subset X_{\rho}$ is said to be $\rho$-convergent to $x \in X_{\rho}$ if $\lim _{n \rightarrow \infty} \rho\left(x_{n}-x\right)=0$.

(ii) A sequence $\left\{x_{n}\right\}_{n \in \mathbb{N}} \subset X_{\rho}$ is said to be $\rho$-Cauchy if $\lim _{n, m \rightarrow \infty} \rho\left(x_{n}-x_{n+m}\right)=0$.

(iii) $X_{\rho}$ is said to be $\rho$-complete if any $\rho$-Cauchy sequence is $\rho$-convergent.

Definition 2.14 The modular $\rho$ has the Fatou property if, for every $y \in X_{\rho}$, we have

$$
\rho(x-y) \leq \liminf _{n \rightarrow \infty} \rho\left(x_{n}-y\right)
$$

whenever $\left\{x_{n}\right\}_{n \in \mathbb{N}} \subset X_{\rho}$ is $\rho$-convergent to $x \in X_{\rho}$.

Let $(X, \rho)$ be a modular space. Define the mapping $D_{\rho}: X_{\rho} \times X_{\rho} \rightarrow[0,+\infty]$ by

$$
D_{\rho}(x, y)=\rho(x-y)
$$

for every $(x, y) \in X \times X$.

We have the following result.

Proposition 2.15 If $\rho$ has the Fatou property, then $D_{\rho}$ is a generalized metric on $X_{\rho}$.

Proof We have just to proof that $D_{\rho}$ satisfies the property $\left(\mathcal{D}_{3}\right)$. Let $x \in X_{\rho}$ and $\left\{x_{n}\right\} \in$ $C\left(D_{\rho}, X_{\rho}, x\right)$, which means that

$$
\lim _{n \rightarrow \infty} \rho\left(x_{n}-x\right)=0 .
$$

Using the Fatou property, for all $y \in X_{\rho}$, we have

$$
\rho(x-y) \leq \liminf _{n \rightarrow \infty} \rho\left(x_{n}-y\right)
$$


which yields

$$
D_{\rho}(x, y) \leq \liminf _{n \rightarrow \infty} D_{\rho}\left(x_{n}, y\right) \leq \limsup _{n \rightarrow \infty} D_{\rho}\left(x_{n}, y\right)
$$

Then $\left(\mathcal{D}_{3}\right)$ is satisfied with $C=1$ and $D_{\rho}$ is a generalized metric on $X_{\rho}$.

The following result is immediate.

Proposition 2.16 Let $\rho$ be a modular on $X$ having the Fatou property. Then

(i) $\left\{x_{n}\right\} \subset X_{\rho}$ is $\rho$-convergent to $x \in X_{\rho}$ if and only if $\left\{x_{n}\right\}$ is $D_{\rho}$-convergent to $x$;

(ii) $\left\{x_{n}\right\} \subset X_{\rho}$ is $\rho$-Cauchy if and only if $\left\{x_{n}\right\}$ is $D_{\rho}$-Cauchy;

(iii) $\left(X_{\rho}, \rho\right)$ is $\rho$-complete if and only if $\left(X_{\rho}, D_{\rho}\right)$ is $D_{\rho}$-complete.

\section{The Banach contraction principle in a generalized metric space}

In this section, we present an extension of the Banach contraction principle to the setting of generalized metric spaces introduced previously.

Let $(X, \mathcal{D})$ be a generalized metric space and $f: X \rightarrow X$ be a mapping.

Definition 3.1 Let $k \in(0,1)$. We say that $f$ is a $k$-contraction if

$$
\mathcal{D}(f(x), f(y)) \leq k \mathcal{D}(x, y), \quad \text { for every }(x, y) \in X \times X .
$$

First, we have the following observation.

Proposition 3.2 Suppose that $f$ is a $k$-contraction for some $k \in(0,1)$. Then any fixed point $\omega \in X$ off satisfies

$$
\mathcal{D}(\omega, \omega)<\infty \quad \Longrightarrow \quad \mathcal{D}(\omega, \omega)=0 .
$$

Proof Let $\omega \in X$ be a fixed point of $f$ such that $\mathcal{D}(\omega, \omega)<\infty$. Since $f$ is a $k$-contraction, we have

$$
\mathcal{D}(\omega, \omega)=\mathcal{D}(f(\omega), f(\omega)) \leq k \mathcal{D}(\omega, \omega)
$$

which implies that $\mathcal{D}(\omega, \omega)=0$ since $k \in(0,1)$ and $\mathcal{D}(\omega, \omega)<\infty$.

For every $x \in X$, let

$$
\delta(\mathcal{D}, f, x)=\sup \left\{\mathcal{D}\left(f^{i}(x), f^{j}(x)\right): i, j \in \mathbb{N}\right\}
$$

We have the following extension of the Banach contraction principle.

Theorem 3.3 Suppose that the following conditions hold:

(i) $(X, \mathcal{D})$ is complete;

(ii) $f$ is a $k$-contraction for some $k \in(0,1)$;

(iii) there exists $x_{0} \in X$ such that $\delta\left(\mathcal{D}, f, x_{0}\right)<\infty$. 
Then $\left\{f^{n}\left(x_{0}\right)\right\}$ converges to $\omega \in X$, a fixed point of $f$. Moreover, if $\omega^{\prime} \in X$ is another fixed point off such that $\mathcal{D}\left(\omega, \omega^{\prime}\right)<\infty$, then $\omega=\omega^{\prime}$.

Proof Let $n \in \mathbb{N}(n \geq 1)$. Since $f$ is a $k$-contraction, for all $i, j \in \mathbb{N}$, we have

$$
\mathcal{D}\left(f^{n+i}\left(x_{0}\right), f^{n+j}\left(x_{0}\right)\right) \leq k \mathcal{D}\left(f^{n-1+i}\left(x_{0}\right), f^{n-1+j}\left(x_{0}\right)\right),
$$

which implies that

$$
\delta\left(\mathcal{D}, f, f^{n}\left(x_{0}\right)\right) \leq k \delta\left(\mathcal{D}, f, f^{n-1}\left(x_{0}\right)\right)
$$

Then, for every $n \in \mathbb{N}$, we have

$$
\delta\left(\mathcal{D}, f, f^{n}\left(x_{0}\right)\right) \leq k^{n} \delta\left(\mathcal{D}, f, x_{0}\right) .
$$

Using the above inequality, for every $n, m \in \mathbb{N}$, we have

$$
\mathcal{D}\left(f^{n}\left(x_{0}\right), f^{n+m}\left(x_{0}\right)\right) \leq \delta\left(\mathcal{D}, f, f^{n}\left(x_{0}\right)\right) \leq k^{n} \delta\left(\mathcal{D}, f, x_{0}\right) .
$$

Since $\delta\left(\mathcal{D}, f, x_{0}\right)<\infty$ and $k \in(0,1)$, we obtain

$$
\lim _{n, m \rightarrow \infty} \mathcal{D}\left(f^{n}\left(x_{0}\right), f^{n+m}\left(x_{0}\right)\right)=0
$$

which implies that $\left\{f^{n}\left(x_{0}\right)\right\}$ is a $\mathcal{D}$-Cauchy sequence.

Since $(X, \mathcal{D})$ is $\mathcal{D}$-complete, there exists some $\omega \in X$ such that $\left\{f^{n}\left(x_{0}\right)\right\}$ is $\mathcal{D}$-convergent to $\omega$.

On the other hand, since $f$ is a $k$-contraction, for all $n \in \mathbb{N}$, we have

$$
\mathcal{D}\left(f^{n+1}\left(x_{0}\right), f(\omega)\right) \leq k \mathcal{D}\left(f^{n}\left(x_{0}\right), \omega\right) .
$$

Letting $n \rightarrow \infty$ in the above inequality, we get

$$
\lim _{n \rightarrow \infty} \mathcal{D}\left(f^{n+1}\left(x_{0}\right), f(\omega)\right)=0
$$

Then $\left\{f^{n}\left(x_{0}\right)\right\}$ is $\mathcal{D}$-convergent to $f(\omega)$. By the uniqueness of the limit (see Proposition 2.4), we get $\omega=f(\omega)$, that is, $\omega$ is a fixed point of $f$.

Now, suppose that $\omega^{\prime} \in X$ is a fixed point of $f$ such that $\mathcal{D}\left(\omega, \omega^{\prime}\right)<\infty$. Since $f$ is a $k$-contraction, we have

$$
\mathcal{D}\left(\omega, \omega^{\prime}\right)=\mathcal{D}\left(f(\omega), f\left(\omega^{\prime}\right)\right) \leq k \mathcal{D}\left(\omega, \omega^{\prime}\right)
$$

which implies by the property $\left(\mathcal{D}_{1}\right)$ that $\omega=\omega^{\prime}$.

Observe that we can replace condition (iii) in Theorem 3.3 by

(iii) $)^{\prime}$ there exists $x_{0} \in X$ such that $\sup \left\{\mathcal{D}\left(x_{0}, f^{r}\left(x_{0}\right)\right): r \in \mathbb{N}\right\}<\infty$. 
In fact, since $f$ is a $k$-contraction, we obtain easily that

$$
\delta\left(\mathcal{D}, f, x_{0}\right) \leq \sup \left\{\mathcal{D}\left(x_{0}, f^{r}\left(x_{0}\right)\right): r \in \mathbb{N}\right\} .
$$

So condition (iii)' implies condition (iii).

The following result (see Kirk and Shahzad [6]) is an immediate consequence of Proposition 2.8 and Theorem 3.3.

Corollary 3.4 Let $(X, d)$ be a complete $b$-metric space and $f: X \rightarrow X$ be a mapping. Suppose that for some $k \in(0,1)$, we have

$$
d(f(x), f(y)) \leq k d(x, y), \quad \text { for every }(x, y) \in X \times X .
$$

If there exists $x_{0} \in X$ such that

$$
\sup \left\{d\left(f^{i}\left(x_{0}\right), f^{j}\left(x_{0}\right)\right): i, j \in \mathbb{N}\right\}<\infty
$$

then the sequence $\left\{f^{n}\left(x_{0}\right)\right\}$ converges to a fixed point of $f$. Moreover, $f$ has one and only one fixed point.

Note that in [1], there is a better result than this given by Corollary 3.4.

The next result is an immediate consequence of Proposition 2.10 and Theorem 3.3.

Corollary 3.5 Let $(X, d)$ be a complete dislocated metric space and $f: X \rightarrow X$ be a mapping. Suppose that for some $k \in(0,1)$, we have

$$
d(f(x), f(y)) \leq k d(x, y), \quad \text { for every }(x, y) \in X \times X .
$$

If there exists $x_{0} \in X$ such that

$$
\sup \left\{d\left(f^{i}\left(x_{0}\right), f^{j}\left(x_{0}\right)\right): i, j \in \mathbb{N}\right\}<\infty
$$

then the sequence $\left\{f^{n}\left(x_{0}\right)\right\}$ converges to a fixed point of $f$. Moreover, $f$ has one and only one fixed point.

The following result is an immediate consequence of Proposition 2.15, Proposition 2.16, and Theorem 3.3.

Corollary 3.6 Let $\left(X_{\rho}, \rho\right)$ be a complete modular space and $f: X \rightarrow X$ be a mapping. Suppose that for some $k \in(0,1)$, we have

$$
\rho(f(x)-f(y)) \leq k \rho(x-y), \quad \text { for every }(x, y) \in X_{\rho} \times X_{\rho} .
$$

Suppose also that $\rho$ satisfies the Fatou property. If there exists $x_{0} \in X_{\rho}$ such that

$$
\sup \left\{\rho\left(f^{i}\left(x_{0}\right)-f^{j}\left(x_{0}\right)\right): i, j \in \mathbb{N}\right\}<\infty
$$

then the sequence $\left\{f^{n}\left(x_{0}\right)\right\} \rho$-converges to some $\omega \in X_{\rho}$, a fixed point off. Moreover, if $\omega^{\prime} \in$ $X_{\rho}$ is another fixed point off such that $\rho\left(\omega-\omega^{\prime}\right)<\infty$, then $\omega=\omega^{\prime}$. 
Observe that in Corollary 3.6, no $\Delta_{2}$-condition is supposed.

\section{4 Ćirić's quasicontraction in a generalized metric space}

In this section, we extend Ćirić's fixed point theorem for quasicontraction type mappings [21] in the setting of generalized metric spaces.

Let $(X, \mathcal{D})$ be a generalized metric space and $f: X \rightarrow X$ be a mapping.

Definition 4.1 Let $k \in(0,1)$. We say that $f$ is a $k$-quasicontraction if

$$
\mathcal{D}(f(x), f(y)) \leq k \max \{\mathcal{D}(x, y), \mathcal{D}(x, T x), \mathcal{D}(y, T y), \mathcal{D}(x, T y), \mathcal{D}(y, T x)\}
$$

for every $(x, y) \in X \times X$.

Proposition 4.2 Suppose that $f$ is a $k$-quasicontraction for some $k \in(0,1)$. Then any fixed point $\omega \in X$ off satisfies

$$
\mathcal{D}(\omega, \omega)<\infty \quad \Longrightarrow \quad \mathcal{D}(\omega, \omega)=0 .
$$

Proof Let $\omega \in X$ be a fixed point of $f$ such that $\mathcal{D}(\omega, \omega)<\infty$. Since $f$ is a $k$-quasicontraction, we have

$$
\mathcal{D}(\omega, \omega)=\mathcal{D}(f(\omega), f(\omega)) \leq k \mathcal{D}(\omega, \omega)
$$

Since $k \in(0,1)$, we get $\mathcal{D}(\omega, \omega)=0$.

We have the following result.

Theorem 4.3 Suppose that the following conditions hold:

(i) $(X, \mathcal{D})$ is complete;

(ii) $f$ is a k-quasicontraction for some $k \in(0,1)$;

(iii) there exists $x_{0} \in X$ such that $\delta\left(\mathcal{D}, f, x_{0}\right)<\infty$.

Then $\left\{f^{n}\left(x_{0}\right)\right\}$ converges to some $\omega \in X$. If $\mathcal{D}\left(x_{0}, f(\omega)\right)<\infty$ and $\mathcal{D}(\omega, f(\omega))<\infty$, then $\omega$ is a fixed point off. Moreover, if $\omega^{\prime} \in X$ is another fixed point off such that $\mathcal{D}\left(\omega, \omega^{\prime}\right)<\infty$ and $\mathcal{D}\left(\omega^{\prime}, \omega^{\prime}\right)<\infty$, then $\omega=\omega^{\prime}$.

Proof Let $n \in \mathbb{N}(n \geq 1)$. Since $f$ is a $k$-quasicontraction, for all $i, j \in \mathbb{N}$, we have

$$
\begin{aligned}
\mathcal{D}\left(f^{n+i}\left(x_{0}\right), f^{n+j}\left(x_{0}\right)\right) \leq & k \max \left\{\mathcal{D}\left(f^{n-1+i}\left(x_{0}\right), f^{n-1+j}\left(x_{0}\right)\right), \mathcal{D}\left(f^{n-1+i}\left(x_{0}\right), f^{n+i}\left(x_{0}\right)\right),\right. \\
& \mathcal{D}\left(f^{n-1+i}\left(x_{0}\right), f^{n+j}\left(x_{0}\right)\right), \mathcal{D}\left(f^{n-1+j}\left(x_{0}\right), f^{n+j}\left(x_{0}\right)\right), \\
& \left.\mathcal{D}\left(f^{n-1+j}\left(x_{0}\right), f^{n+i}\left(x_{0}\right)\right)\right\},
\end{aligned}
$$

which implies that

$$
\delta\left(\mathcal{D}, f, f^{n}\left(x_{0}\right)\right) \leq k \delta\left(\mathcal{D}, f, f^{n-1}\left(x_{0}\right)\right)
$$

Hence, for any $n \geq 1$, we have

$$
\delta\left(\mathcal{D}, f, f^{n}\left(x_{0}\right)\right) \leq k^{n} \delta\left(\mathcal{D}, f, x_{0}\right) .
$$


Using the above inequality, for every $n, m \in \mathbb{N}$, we have

$$
\mathcal{D}\left(f^{n}\left(x_{0}\right), f^{n+m}\left(x_{0}\right)\right) \leq \delta\left(\mathcal{D}, f, f^{n}\left(x_{0}\right)\right) \leq k^{n} \delta\left(\mathcal{D}, f, x_{0}\right)
$$

Since $\delta\left(\mathcal{D}, f, x_{0}\right)<\infty$ and $k \in(0,1)$, we obtain

$$
\lim _{n, m \rightarrow \infty} \mathcal{D}\left(f^{n}\left(x_{0}\right), f^{n+m}\left(x_{0}\right)\right)=0
$$

which implies that $\left\{f^{n}\left(x_{0}\right)\right\}$ is a $\mathcal{D}$-Cauchy sequence.

Since $(X, \mathcal{D})$ is $\mathcal{D}$-complete, there exists some $\omega \in X$ such that $\left\{f^{n}\left(x_{0}\right)\right\}$ is $\mathcal{D}$-convergent to $\omega$.

Now, we suppose that $\mathcal{D}\left(x_{0}, f(\omega)\right)<\infty$. Using the inequality

$$
\mathcal{D}\left(f^{n}\left(x_{0}\right), f^{n+m}\left(x_{0}\right)\right) \leq k^{n} \delta\left(\mathcal{D}, f, x_{0}\right)
$$

for every $n, m \in \mathbb{N}$, by the property $\left(\mathcal{D}_{3}\right)$, there exists some constant $C>0$ such that

$$
\mathcal{D}\left(\omega, f^{n}\left(x_{0}\right)\right) \leq C \limsup _{m \rightarrow \infty} \mathcal{D}\left(f^{n}\left(x_{0}\right), f^{n+m}\left(x_{0}\right)\right) \leq C k^{n} \delta\left(\mathcal{D}, f, x_{0}\right)
$$

for every $n \in \mathbb{N}$.

On the other hand, we have

$$
\mathcal{D}\left(f\left(x_{0}\right), f(\omega)\right) \leq k \max \left\{\mathcal{D}\left(x_{0}, \omega\right), \mathcal{D}\left(x_{0}, f\left(x_{0}\right)\right), \mathcal{D}(\omega, f(\omega)), \mathcal{D}\left(f\left(x_{0}\right), \omega\right), \mathcal{D}\left(x_{0}, f(\omega)\right)\right\}
$$

Using (4.1) and (4.2), we get

$$
\mathcal{D}\left(f\left(x_{0}\right), f(\omega)\right) \leq \max \left\{k C \delta\left(\mathcal{D}, f, x_{0}\right), k \delta\left(\mathcal{D}, f, x_{0}\right), k \mathcal{D}(\omega, f(\omega)), k \mathcal{D}\left(x_{0}, f(\omega)\right)\right\}
$$

Again, using the above inequality, we have

$$
\mathcal{D}\left(f^{2}\left(x_{0}\right), f(\omega)\right) \leq \max \left\{k^{2} C \delta\left(\mathcal{D}, f, x_{0}\right), k^{2} \delta\left(\mathcal{D}, f, x_{0}\right), k \mathcal{D}(\omega, f(\omega)), k^{2} \mathcal{D}\left(x_{0}, f(\omega)\right)\right\}
$$

Continuing this process, by induction we get

$$
\mathcal{D}\left(f^{n}\left(x_{0}\right), f(\omega)\right) \leq \max \left\{k^{n} C \delta\left(\mathcal{D}, f, x_{0}\right), k^{n} \delta\left(\mathcal{D}, f, x_{0}\right), k \mathcal{D}(\omega, f(\omega)), k^{n} \mathcal{D}\left(x_{0}, f(\omega)\right)\right\},
$$

for every $n \geq 1$. Therefore, we have

$$
\limsup _{n \rightarrow \infty} \mathcal{D}\left(f^{n}\left(x_{0}\right), f(\omega)\right) \leq k \mathcal{D}(\omega, f(\omega))
$$

since $\mathcal{D}\left(x_{0}, f(\omega)\right)<\infty$ and $\delta\left(\mathcal{D}, f, x_{0}\right)<\infty$. Using the property $\left(\mathcal{D}_{3}\right)$, we get

$$
\mathcal{D}(f(\omega), \omega) \leq \limsup _{n \rightarrow \infty} \mathcal{D}\left(f^{n}\left(x_{0}\right), f(\omega)\right) \leq k \mathcal{D}(\omega, f(\omega))
$$

which implies that $\mathcal{D}(f(\omega), \omega)=0$ since $\mathcal{D}(\omega, f(\omega))<\infty$ and $k \in(0,1)$. Then $\omega$ is a fixed point of $f$. By Proposition 4.2, we have $\mathcal{D}(\omega, \omega)=0$. 
Finally, suppose that $\omega^{\prime} \in X$ is another fixed point of $f$ such that $\mathcal{D}\left(\omega, \omega^{\prime}\right)<\infty$ and $\mathcal{D}\left(\omega^{\prime}, \omega^{\prime}\right)<\infty$. By Proposition 4.2, we have $\mathcal{D}\left(\omega^{\prime}, \omega^{\prime}\right)=0$. Since $f$ is a $k$-quasicontraction, we get

$$
\mathcal{D}\left(\omega, \omega^{\prime}\right)=\mathcal{D}\left(f(\omega), f(\omega)^{\prime}\right) \leq k \mathcal{D}\left(\omega, \omega^{\prime}\right),
$$

which implies that $\omega=\omega^{\prime}$.

As in the previous section, from Theorem 4.3, we can obtain fixed point results for Ćirić's quasicontraction type mappings in various spaces including standard metric spaces, $b$-metric spaces, dislocated metric spaces, and modular spaces.

\section{Banach contraction principle in a generalized metric space with a partial order}

In this section, we extend the Banach contraction principle to the class of generalized metric spaces with a partial order.

Let $(X, \mathcal{D})$ be a generalized metric space and $f: X \rightarrow X$ be a mapping. Let $\preceq$ be a partial order on $X$. We denote by $E_{\leq}$the subset of $X \times X$ defined by

$$
E_{\preceq}=\{(x, y) \in X \times X: x \preceq y\} .
$$

Now, let us introduce some concepts.

Definition 5.1 We say that $f$ is weak continuous if the following condition holds: if $\left\{x_{n}\right\} \subset$ $X$ is $\mathcal{D}$-convergent to $x \in X$, then there exists a subsequence $\left\{x_{n_{q}}\right\}$ of $\left\{x_{n}\right\}$ such that $\left\{f\left(x_{n_{q}}\right)\right\}$ is $\mathcal{D}$-convergent to $f(x)($ as $q \rightarrow \infty)$.

Definition 5.2 We say that $f$ is $\preceq$-monotone if the following condition holds:

$$
(x, y) \in E_{\leq} \quad \Longrightarrow \quad(f(x), f(y)) \in E_{\preceq} .
$$

Definition 5.3 We say that the pair $(X, \preceq)$ is $\mathcal{D}$-regular if the following condition holds: for every sequence $\left\{x_{n}\right\} \subset X$ satisfying $\left(x_{n}, x_{n+1}\right) \in E_{\leq}$, for every $n$ large enough, if $\left\{x_{n}\right\}$ is $\mathcal{D}$-convergent to $x \in X$, then there exists a subsequence $\left\{x_{n_{q}}\right\}$ of $\left\{x_{n}\right\}$ such that $\left(x_{n_{q}}, x\right) \in E_{\leq}$, for every $q$ large enough.

Definition 5.4 We say that $f$ is a weak $k$-contraction for some $k \in(0,1)$ if the following condition holds:

$$
(x, y) \in E_{\leq} \quad \Longrightarrow \quad \mathcal{D}(f(x), f(y)) \leq k \mathcal{D}(x, y) .
$$

Our first result holds under the weak continuity assumption.

Theorem 5.5 Suppose that the following conditions hold:

(i) $(X, \mathcal{D})$ is complete;

(ii) $f$ is weak continuous;

(iii) $f$ is a weak $k$-contraction for some $k \in(0,1)$; 
(iv) there exists $x_{0} \in X$ such that $\delta\left(\mathcal{D}, f, x_{0}\right)<\infty$ and $\left(x_{0}, f\left(x_{0}\right)\right) \in E_{\leq}$;

(v) $f$ is $\preceq$-monotone.

Then $\left\{f^{n}\left(x_{0}\right)\right\}$ converges to some $\omega \in X$ such that $\omega$ is a fixed point of $f$. Moreover, if $\mathcal{D}(\omega, \omega)<\infty$, then $\mathcal{D}(\omega, \omega)=0$.

Proof Since $f$ is $\preceq$-monotone and $\left(x_{0}, f\left(x_{0}\right)\right) \in E_{\preceq}$, then

$$
\left(f^{n}\left(x_{0}\right), f^{n+1}\left(x_{0}\right)\right) \in E_{\leq}, \quad \text { for every } n \in \mathbb{N} .
$$

Since $\preceq$ is a partial order (so it is transitive), then

$$
(p, q) \in \mathbb{N} \times \mathbb{N}, \quad p \leq q \quad \Longrightarrow \quad f^{p}\left(x_{0}\right) \preceq f^{q}\left(x_{0}\right) .
$$

Let $n \in \mathbb{N}(n \geq 1)$. Since $f$ is a weak $k$-contraction and $\mathcal{D}$ is symmetric, for all $i, j \in \mathbb{N}$, we have

$$
\mathcal{D}\left(f^{n+i}\left(x_{0}\right), f^{n+j}\left(x_{0}\right)\right) \leq k \mathcal{D}\left(f^{n-1+i}\left(x_{0}\right), f^{n-1+j}\left(x_{0}\right)\right),
$$

which implies that

$$
\delta\left(\mathcal{D}, f, f^{n}\left(x_{0}\right)\right) \leq k \delta\left(\mathcal{D}, f, f^{n-1}\left(x_{0}\right)\right)
$$

Then, for every $n \in \mathbb{N}$, we have

$$
\delta\left(\mathcal{D}, f, f^{n}\left(x_{0}\right)\right) \leq k^{n} \delta\left(\mathcal{D}, f, x_{0}\right)
$$

Using the above inequality, for every $n, m \in \mathbb{N}$, we have

$$
\mathcal{D}\left(f^{n}\left(x_{0}\right), f^{n+m}\left(x_{0}\right)\right) \leq \delta\left(\mathcal{D}, f, f^{n}\left(x_{0}\right)\right) \leq k^{n} \delta\left(\mathcal{D}, f, x_{0}\right) .
$$

Since $\delta\left(\mathcal{D}, f, x_{0}\right)<\infty$ and $k \in(0,1)$, we obtain

$$
\lim _{n, m \rightarrow \infty} \mathcal{D}\left(f^{n}\left(x_{0}\right), f^{n+m}\left(x_{0}\right)\right)=0
$$

which implies that $\left\{f^{n}\left(x_{0}\right)\right\}$ is a $\mathcal{D}$-Cauchy sequence.

Since $(X, \mathcal{D})$ is $\mathcal{D}$-complete, there exists some $\omega \in X$ such that $\left\{f^{n}\left(x_{0}\right)\right\}$ is $\mathcal{D}$-convergent to $\omega$. Since $f$ is weak continuous, there exists a subsequence $\left\{f^{n_{q}}\left(x_{0}\right)\right\}$ of $\left\{f^{n}\left(x_{0}\right)\right\}$ such that $\left\{f^{n_{q}+1}\left(x_{0}\right)\right\}$ is $\mathcal{D}$-convergent to $f(\omega)$ (as $q \rightarrow \infty$ ). By the uniqueness of the limit, we get $\omega=f(\omega)$, that is, $\omega$ is a fixed point of $f$.

Suppose now that $\mathcal{D}(\omega, \omega)<\infty$, since $(\omega, \omega) \in E_{\preceq}$, we have

$$
\mathcal{D}(\omega, \omega)=\mathcal{D}(f(\omega), f(\omega)) \leq k \mathcal{D}(\omega, \omega)
$$

which implies that $\mathcal{D}(\omega, \omega)=0$ (since $k \in(0,1)$ ).

Remark 5.6 Theorem 5.5 is an extension of Ran and Reurings fixed point result [22] established in the setting of metric spaces under the continuity of the mapping $f$. 
Now, we replace the weak continuity assumption by the $\mathcal{D}$-regularity of the pair $(X, \preceq)$. We have the following result.

Theorem 5.7 Suppose that the following conditions hold:

(i) $(X, \mathcal{D})$ is complete;

(ii) $(X, \preceq)$ is $\mathcal{D}$-regular;

(iii) $f$ is a weak $k$-contraction for some $k \in(0,1)$;

(iv) there exists $x_{0} \in X$ such that $\delta\left(\mathcal{D}, f, x_{0}\right)<\infty$ and $\left(x_{0}, f\left(x_{0}\right)\right) \in E_{\leq}$;

(v) $f$ is $\preceq$-monotone.

Then $\left\{f^{n}\left(x_{0}\right)\right\}$ converges to some $\omega \in X$ such that $\omega$ is a fixed point of $f$. Moreover, if $\mathcal{D}(\omega, \omega)<\infty$, then $\mathcal{D}(\omega, \omega)=0$.

Proof Following the proof of the previous theorem, we know that $\left\{f^{n}\left(x_{0}\right)\right\}$ is $\mathcal{D}$-convergent to some $\omega \in X$ and

$$
\left(f^{n}\left(x_{0}\right), f^{n+1}\left(x_{0}\right)\right) \in E_{\preceq}, \quad \text { for every } n \in \mathbb{N} .
$$

Since $(X, \preceq)$ is $\mathcal{D}$-regular, there exists a subsequence $\left\{f^{n_{q}}\left(x_{0}\right)\right\}$ of $\left\{f^{n}\left(x_{0}\right)\right\}$ such that $\left(f^{n_{q}}\left(x_{0}\right), \omega\right) \in E_{\leq}$, for every $q$ large enough. On the other hand, $f$ is a weak $k$-contraction, so we have

$$
\mathcal{D}\left(f^{n_{q}+1}\left(x_{0}\right), f(\omega)\right) \leq k \mathcal{D}\left(f^{n_{q}}\left(x_{0}\right), \omega\right),
$$

for every $q$ large enough. Letting $q \rightarrow \infty$ in the above inequality, we get

$$
\lim _{q \rightarrow \infty} \mathcal{D}\left(f^{n_{q}+1}\left(x_{0}\right), f(\omega)\right)=0
$$

which implies that $\left\{f^{n_{q}+1}\left(x_{0}\right)\right\}$ is $\mathcal{D}$-convergent to $f(\omega)$. By uniqueness of the limit, we get $f(\omega)=\omega$.

Similar to the proof in the previous theorem, we have $\mathcal{D}(\omega, \omega)=0$.

Remark 5.8 Theorem 5.7 is an extension of Nieto and Rodíguez-López fixed point result ([23], Theorem 4) established in the setting of metric spaces.

Competing interests

The authors declare that they have no competing interests.

\section{Authors' contributions}

The authors contributed equally and significantly in writing this paper. Both authors read and approved the final manuscript.

\section{Acknowledgements}

This project was funded by the National Plan for Science, Technology and Innovation (MAARIFAH), King Abdulaziz City for Science and Technology, Kingdom of Saudi Arabia, Award Number (12-MAT 2895-02).

Received: 9 December 2014 Accepted: 20 April 2015 Published online: 29 April 2015

\section{References}

1. Czerwik, S: Contraction mappings in b-metric spaces. Acta Math. Inform. Univ. Ostrav. 1, 5-11 (1993)

2. Akkouchi, M: Common fixed point theorems for two self mappings of a $b$-metric space under an implicit relation. Hacet. J. Math. Stat. 40(6), 805-810 (2011)

3. Berinde, V: Sequences of operators and fixed points in quasimetric spaces. Stud. Univ. Babeş-Bolyai, Math. 41, 23-27 (1996) 
4. Boriceanu, M, Bota, M, Petrusel, A: Multivalued fractals in b-metric spaces. Cent. Eur. J. Math. 8(2), 367-377 (2010)

5. Czerwik, S, Dlutek, K, Singh, SL: Round-off stability of iteration procedures for set-valued operators in $b$-metric spaces. J. Natur. Phys. Sci. 11, 87-94 (2007)

6. Kirk, W, Shahzad, N: b-Metric spaces. In: Fixed Point Theory in Distance Spaces, pp. 113-131. Springer, Berlin (2014)

7. Popovic, B, Radenovic, S, Shukla, S: Fixed point results to tvs-cone b-metric spaces. Gulf J. Math. 1, 51-64 (2013)

8. Hitzler, P, Seda, AK: Dislocated topologies. J. Electr. Eng. 51(12), 3-7 (2000)

9. Aage, CT, Salunke, JN: The results on fixed points in dislocated and dislocated quasi-metric space. Appl. Math. Sci. 2(59), 2941-2948 (2008)

10. Ahamad, MA, Zeyada, FM, Hasan, GF: Fixed point theorems in generalized types of dislocated metric spaces and its applications. Thai J. Math. 11, 67-73 (2013)

11. Hitzler, P: Generalized metrics and topology in logic programming semantics. Dissertation, Faculty of Science, National University of Ireland, University College, Cork (2001)

12. Karapinar, E, Salimi, P: Dislocated metric space to metric spaces with some fixed point theorems. Fixed Point Theory Appl. 2013, 222 (2013)

13. Nakano, H: Modular semi-ordered spaces, Tokyo, Japan (1959)

14. Musielak, J, Orlicz, W: On modular spaces. Stud. Math. 18, 49-65 (1959)

15. Dominguez Benavides, T, Khamsi, MA, Samadi, S: Uniformly Lipschitzian mappings in modular function spaces. Nonlinear Anal. 46(2), 267-278 (2001)

16. Hajji, A, Hanebaly, E: Fixed point theorem and its application to perturbed integral equations in modular function spaces. Electron. J. Differ. Equ. 2005, 105 (2005)

17. Khamsi, MA: Nonlinear semigroups in modular function spaces. Math. Jpn. 37, 291-299 (1992)

18. Khamsi, MA, Kozlowski, WM, Reich, S: Fixed point theory in modular function spaces. Nonlinear Anal. 14, 935-953 (1990)

19. Kozlowski, WM: Modular Function Spaces. Monographs and Textbooks in Pure and Applied Mathematics, vol. 122. Dekker, New York (1988)

20. Razani, A, Pour, SH, Nabizadeh, E, Mohamadi, MB: A new version of the Ćirić quasi-contraction principle in the modular space. Novi Sad J. Math. 43(2), 1-9 (2003)

21. Ćirić, LB: A generalization of Banach's contraction principle. Proc. Am. Math. Soc. 45(2), 267-273 (1974)

22. Ran, ACM, Reurings, MCB: A fixed point theorem in partially ordered sets and some applications to matrix equations. Proc. Am. Math. Soc. 132(5), 1435-1443 (2004)

23. Nieto, JJ, Rodríquez-López, R: Existence and uniqueness of fixed point in partially ordered sets and applications to ordinary differential equations. Acta Math. Sin. 23(12), 2205-2212 (2007)

\section{Submit your manuscript to a SpringerOpen ${ }^{\circ}$ journal and benefit from:}

- Convenient online submission

Rigorous peer review

- Immediate publication on acceptance

- Open access: articles freely available online

- High visibility within the field

- Retaining the copyright to your article 